\title{
Fuzzy uncertainty representations of co-seismic displacement measurements issued from SAR imagery
}

\author{
Yajing Yan, Gilles Mauris, Member, IEEE, Emmanuel Trouvé, Senior Member, IEEE, Virginie Pinel
}

\begin{abstract}
An emerging way to reduce the geodetic parameter uncertainty is to combine the large numbers of data provided by satellite synthetic aperture radar (SAR) images. However, the measurements by radar imagery are subject to both random and systematic uncertainties. Thus, mathematical theories which are adequate for each type of uncertainty representation and handling have to be selected. Probability theory is known as the adequate theory for uncertainties corresponding to random variables, but questionable for systematic uncertainties, arising from information incompleteness. Fuzzy theory being a generalization of interval mathematics, is more adapted to such uncertainty. Moreover it provides a bridge with probability theory by its ability to represent a family of probability distributions. Therefore, we consider here the conventional probability and the fuzzy approaches for handling the random and systematic uncertainties of D-InSAR and SAR amplitude image correlation measurements. The applications are performed on the measurement of the displacement field due to the 2005 Kashmir earthquake. The fuzzy approach, being free from distribution and independence hypotheses, gives the most pessimistic uncertainty assessment, while the conventional probability approach gives the most optimistic uncertainty assessment. As confirmed by the Monte-Carlo simulation applied to an Earth deformation model, the actual uncertainty should be situated between the fuzzy and conventional uncertainties.
\end{abstract}

Index Terms-measurement uncertainty, fuzzy/possibility theory, remote sensing, SAR images, ground displacement, coseismic observations

\section{INTRODUCTION}

Geodetic data, such as satellite images (radar and optic), are important remote sensing sources of information for ground displacement measurement with great accuracy over large area. So far, with the increasing number of operational sensors, large volumes of SAR images acquired in different modes, ascending and descending passes at various incident angles and frequencies, are available. Moreover, the launching in the coming years of the future satellite generation Sentinel will provide a large number of free SAR data [1]. Consequently, using large number of geodetic measurements in order to accurately determine the displacement field is becoming more and more frequent in geophysics, especially to better constraint the geophysical modeling [2] [3] [4] [5]. In this context, one

Laboratoire d'Informatique, Systèmes, Traitement de l'Information et de la Connaissance, Université de Savoie - Polytech AnnecyChambéry, BP80439, F74944, Annecy-le-Vieux Cedex, France. Email: yajing.yan,gilles.mauris,emmanuel.trouve@univ-savoie.fr

ISTerre, CNRS, IRD R219, Université de Savoie, Campus Scientifique, 73376, Le Bourget du Lac Cedex, France. Email: virginie.pinel@univsavoie.fr important purpose of geodetic data processing is to reduce parameter uncertainty by an adequate combination of all the available measurements.

Once gross errors have been eliminated, measurement uncertainties come from random and systematic effects. Concerning the measurements by radar (SAR) imagery, the uncertainties arise from noise sources of radar instrument, on the path of radar wave propagation, at the reflecting surface, as well as uncertainty sources introduced by data processing [4]. On one hand, random uncertainty exists due to decorrelation noise, since there are usually some backscattering property changes on the ground between two subsequent SAR acquisitions. On the other hand, systematic uncertainties can be induced by atmospheric disturbances depending on the state of atmosphere and the ground surface at the time of the two SAR acquisitions. Also, it can result from the imprecision of orbit auxiliary information, Digital Elevation Model (DEM) errors, as well as from the imperfect corrections during data processing, which deviate the data by a constant or a ramp from the true value.

To model such systematic uncertainties coming from limited knowledge (called also epistemic uncertainties in many cases), probability theory is questionable, and thus fuzzy/possibility theory has been proposed in ([6], [7]) and further developed by a few authors in a general measurement context [8] [9] [10] [11], and also by a few authors in geosciences [12] [13] [14] [15]. Thus, it is worthwhile to study the consideration of the most suitable uncertainty theories according to the application specificities. This paper is a first contribution to such issue for the measurement of displacement field by a joint inversion of D-InSAR and SAR amplitude image correlation measurements by a least squares adjustment.

This paper is organized as follows. In section II, the conventional probability approach and the fuzzy approach for uncertainty representation and propagation in the linear case are detailed. The available data in the considered application and their associated uncertainties are described in section III. Then, the two approaches are applied to the measurement of the Earth's surface 3D displacement field due to the 2005 Kashmir earthquake (magnitude of 7.6). The behaviours of each uncertainty approach are highlighted through intercomparisons of results. In section IV, a model based reference of the 3D displacement field is established by using a mechanical Earth deformation model in order to evaluate the results obtained previously. The interest of the fuzzy approach is highlighted with respect to the results provided by the physical model. Finally, some conclusions and perspectives are drawn. 


\section{UNCERTAINTY REPRESENTATION APPROACHES}

In geodetic practice, there are always multiples sources of uncertainty in the considered measurements, which leads to complex characteristics for the associated uncertainties. Probability theory is the adequate theory for uncertainties corresponding to random variables, the latter being described by one probability distribution (often a Gaussian one) or more simply by the first two moments, i.e. the mean and the variance. Systematic uncertainties, arising from information incompleteness, are often described by an interval and thus cannot be associated to one single probability distribution. Fuzzy theory can be considered as a generalization of interval mathematics and provides a bridge with probability theory by its ability to represent a family of probability distributions [16]. Therefore, hereafter, we consider the conventional probability and fuzzy approaches for handling the random and systematic uncertainties encountered in SAR displacement measurements.

\section{A. Conventional probability approach}

The standard reference in uncertainty modeling is the Guide to the Expression of Uncertainty in Measurement (GUM) edited by an international consortium of legal and professional organizations [17]. The GUM groups the occurring uncertain quantities into Type A and Type B. Uncertainties of Type A are determined with the classical statistical methods, while Type B is determined by other ways, e.g. experience and knowledge about an instrument. Both types of uncertainty can have random and systematic components. In fact, the GUM proposes to treat both uncertainties (random and systematic) in a stochastic framework and considers variances to describe them and processes them with the law of propagation of variance, generally assuming independence. Applying this approach to linear inversion by a least squares adjustment, the uncertainties are propagated as follows. Let us consider $U$ a set of $m$ quantities $u_{k}\left(x_{1}, x_{2}, \ldots, x_{n}\right)$ (e.g. 3D (E, N, Up) Earth surface displacement) which are linear combinations of the vector of $n$ measured variables $R=\left(r_{1}, r_{2}, \ldots, r_{n}\right)$ (e.g. projections of the $3 \mathrm{D}$ displacement) with coefficients vector $A=\left(a_{1, k}, a_{2, k}, \ldots, a_{n, k}\right),(k=1, \ldots, m)$. Thus:

$$
u_{k}=\sum_{i=1}^{n} a_{i, k} r_{i}: U=A^{T} R
$$

If the variance-covariance matrix of $R$ is denoted by $\Sigma_{R}$,

$$
\Sigma_{R}=\left(\begin{array}{cccc}
\sigma_{1}^{2} & \sigma_{12} & \sigma_{13} & \cdots \\
\sigma_{21} & \sigma_{2}^{2} & \sigma_{23} & \cdots \\
\sigma_{31} & \sigma_{32} & \sigma_{3}^{2} & \cdots \\
\vdots & \vdots & \vdots & \ddots
\end{array}\right)
$$

then, the variance-covariance matrix $\Sigma_{U}$ of $U$ is given by

$$
\Sigma_{U i, j}=\sum_{k=1}^{n} \sum_{l=1}^{n} a_{i, k} \Sigma_{R k, l} a_{l, j}: \Sigma_{U}=A^{T} \Sigma_{R} A
$$

When $R$ and $U$ are related by a $n \times m$ matrix $P$ which represents the geometrical relationship between the measured variables and the displacement to be determined, the inversion by the generalized least squares method [18] [19] leads to:

$$
A^{T}=\left(P^{T} \Sigma_{R}^{-1} P\right)^{-1} P^{T} \Sigma_{R}^{-1}
$$

thus the nominal value $U$ is given by

$$
U=\left(P^{T} \Sigma_{R}^{-1} P\right)^{-1} P^{T} \Sigma_{R}^{-1} R
$$

and the uncertainty $\Sigma_{U}$ is given by

$$
\Sigma_{U}=\left(P^{T} \Sigma_{R}^{-1} P\right)^{-1}
$$

with $\Sigma_{R}$ the variance-covariance matrix of $R$.

To determine confidence intervals for the parameters, the GUM suggests to use a Gaussian distribution (justified by the central limit theorem), and for other distributions to apply Monte Carlo simulations. This well known approach is fully justified in cases of a lot of data having independent random uncertainties but questionable for systematic uncertainties often dependent and far from a Gaussian representation. Therefore, it generally leads to an over-optimistic assessment of the uncertainties.

\section{B. Fuzzy/possibility approach}

The possibility theory, first introduced by L. Zadeh in 1978 [20], is associated with the theory of fuzzy sets by the semantics of uncertainty that it gives the membership function. A possibility distribution $\pi$ is a mapping from a set to the unit interval such that $\pi(x)=1$ for some $x$ belonging to the set of reals (Fig. 1). A possibility distribution $\pi_{1}$ is called more specific (i.e. thinner in a broad sense) than $\pi_{2}$ as soon as $\forall x \in \Re, \pi_{1}(x) \leq \pi_{2}(x)$ (fuzzy set inclusion). The more specific $\pi$ is, the more informative it is. If for one $x, \pi(x)=1$ and for all $y \neq x, \pi(y)=0$, then $\pi$ is totally specific (fully precise and certain knowledge). If for all $x, \pi(x)=1$, then $\pi$ is totally non specific (complete ignorance) (Fig. 1).

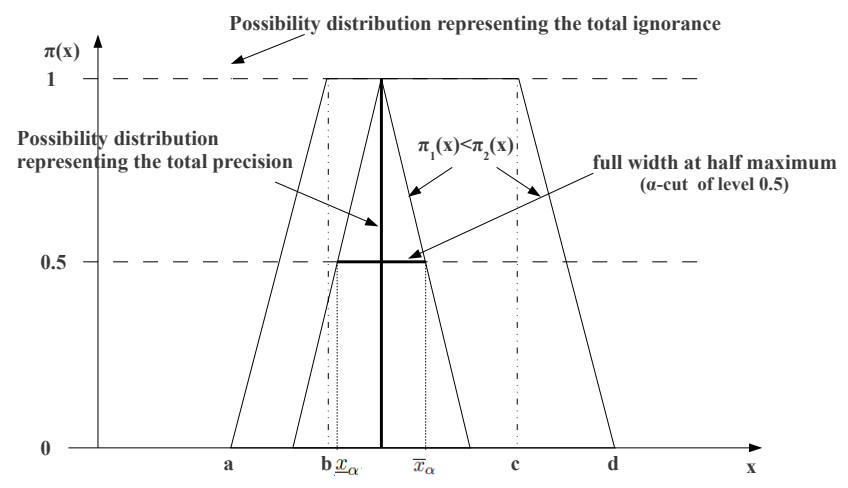

Fig. 1: Example of fuzzy possibility distributions.

In fact, a numerical degree of possibility can be viewed as an upper bound to a probability degree [16]. Namely, with every possibility distribution $\pi$ one can associate a non-empty family of probability measures dominated by the possibility measure: $\mathcal{P}(\pi)=\{P, \forall A, P(A) \leq \Pi(A)\}$. This provides a bridge 
between probability and possibility. There is also a bridge with interval calculus. Indeed, a unimodal numerical possibility distribution may also be viewed as a nested set of coverage intervals, which are the $\alpha$-cuts of $\pi:\left[\underline{x}_{\alpha}, \bar{x}_{\alpha}\right]=\{x, \pi(x) \geq$ $\alpha\}$. Obviously, the confidence intervals built around the same point $x_{0}$ are nested. It has been proven in [21] that stacking the coverage intervals of a probability distribution $F$ on top of one another leads to a possibility distribution (denoted $\pi^{x_{0}}$ having $x_{0}$ as modal value). In fact, in this way, the $\alpha$ cuts of $\pi^{x_{0}}$ are identified with the confidence interval $I_{\beta}^{\star}$ of probability level $\beta=1-\alpha$ around the nominal value $x_{0}$. In this way a probability distribution can be represented by an equivalent possibility distribution. Moreover, a possibility distribution can be used to represent a family of probability distributions by taking the largest $1-\alpha$ confidence intervals obtained from each probability distribution of the family. This is useful to represent uncertainty when only partial probability knowledge is available. For example if the measured variable $r$ is known to be bounded and unimodal (with mode $r_{0}$ ) and with $\sigma_{r}$ as standard deviation, then the maximum specific possibility distribution is a triangular possibility distribution with the mode $r_{0}$ as vertex and with $\left[r_{0}-\sqrt{3} \sigma_{r}, r_{0}+\sqrt{3} \sigma_{r}\right]$ as support [7]. To represent the uncertainty in the fuzzy approach by a single parameter (in a way similar to the variance in the probability approach), the full width at half maximum (denoted $h_{0.5}$ ) of the possibility distribution which corresponds to a $\alpha$-cut level of 0.5 , is commonly used. In summary, a possibility distribution can model both random and systematic uncertainties in a unified modeling. Afterwards, the possibility distributions are propagated in the least squares adjustment using fuzzy arithmetic based on Zadehs extension principle [20]:

$$
U=\left(P^{T} \Sigma_{R}^{-1} P\right)^{-1} P^{T} \Sigma_{R}^{-1} \otimes R
$$

where $\otimes$ is the fuzzy multiplication matrix operation in which the conventional sum and scalar product are replaced by the corresponding fuzzy operations [22].

In this principle, the variables are considered as non interactive variables; this corresponds somehow to consider a total dependence between variables. Consequently, uncertainty propagation by the fuzzy approach leads to an over-pessimistic assessment of the uncertainties.

\section{Displacement uncertainty analysis by the two approaches}

In this article, to combine the available measurements, we apply the Generalized Least Squares (GLS) method [18] [19] which gives the nominal displacement value and its corresponding variance from the variances of the measurements provided by the SAR image processing. For the fuzzy approach, we use the same GLS matrix $A^{T}$ to obtain the forward model. But we build the possibility distributions of the displacement from the value $r_{0}$ and their associated uncertainty $\sigma_{r_{0}}$, considering that $\sigma_{r_{0}}$ contains both random and systematic components. Moreover, the measurements are considered as bounded (this is the case in the considered context), thus we represent them by a symmetric triangular fuzzy distribution with support $\left[r_{0}-\sqrt{3} \sigma_{r_{0}}, r_{0}+\sqrt{3} \sigma_{r_{0}}\right]$. Therefore, the full width at half maximum is $\sqrt{3} \sigma_{r_{0}}$. Let us remark that with a Gaussian assumption of standard deviation $\sigma_{r_{0}}$, the value corresponding to the $0.5 \alpha$-cut the width of the Gauss equivalent possibility distribution is equal to $1.35 \sigma_{r_{0}}$, i.e. the width of the $50 \%$ confidence interval of a Gaussian distribution with $\sigma_{r_{0}}$ as standard deviation.

\section{DISPLACEMENT MEASUREMENTS DUE TO THE 2005 KASHMIR EARTHQUAKE}

\section{A. Description of the available data}

The sub-pixel image correlation and the differential interferometry (D-InSAR) are two conventional techniques used to extract displacement measurements from SAR data. The subpixel image correlation computes the offsets in range (line of sight) and azimuth (along the trajectory of satellite) directions on amplitude images, with a sub-pixel accuracy. It is widely used to measure the displacement of great magnitude [23] [24] [25]. The D-InSAR, using the phase information, provides the displacement in range direction with an uncertainty in the order of centimeter, even millimeter [26] [19]. This technique is usually applied to measure the displacement of small magnitude. In case of a strong earthquake induced by a rupture of a fault, in near field of the fault, the measurements from sub-pixel image correlation can provide reliable displacement information. While in far field of the fault, the measurements from D-InSAR are taken as accurate sources [23].

In this article, a series of co-seismic ENVISAT images from October 2004 to June 2006 are used to map the deformation due to the 2005 Kashmir earthquake. 22 measurements from sub-pixel image correlation and 5 measurements from DInSAR, are available respectively. In near field of the fault, because of coherence loss, phase information cannot be extracted by D-InSAR, thus there is no D-InSAR measurements available in this area. These measurements can be classified in four families according to their acquisition geometry: ascending range (Asc. Rg), ascending azimuth (Asc. Az), descending range (Des. Rg) and descending azimuth (Des. Az). In each family, in first approximation, all the measurements are considered as corresponding to the same displacement (in the same direction) because the incident angle is the same for all the measurements.

For measurements from sub-pixel image correlation, the uncertainty parameter is the so called "pseudo-variance" provided by the ROIPAC software [27]. In fact, this pseudovariance includes both random and systematic components and thus it is not a conventional statistical variance. The random part comes from the decorrelation noise present in the data, while the systematic part comes from the default of correlation method and the DEM error estimation. However, the possible systematic uncertainty due to the imperfect data processing is not included in the pseudo-variance.

For measurements from D-InSAR, the uncertainty parameter corresponds to the variance of the phase value estimated from the coherence [28]. It characterizes only random variations in the phase value. However, systematic uncertainty due to phase unwrapping errors, atmospheric impact, etc., is probably present in the measurement, but has not yet been taken into account in our approach. 


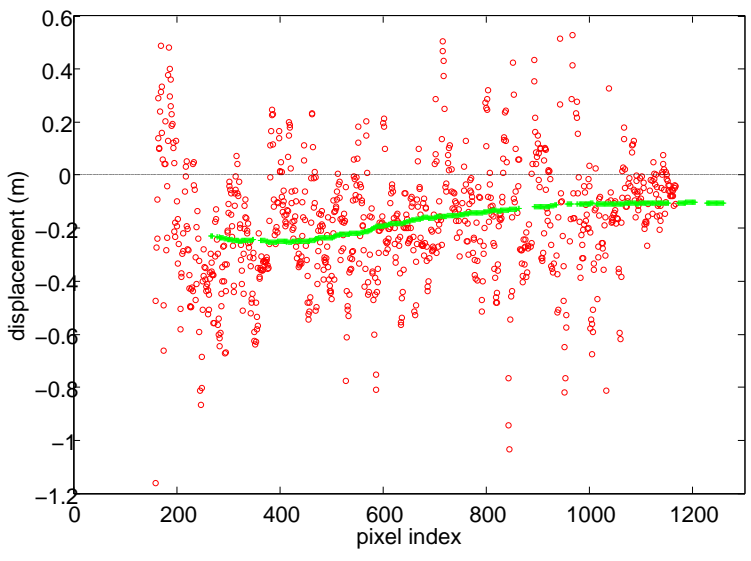

(a)

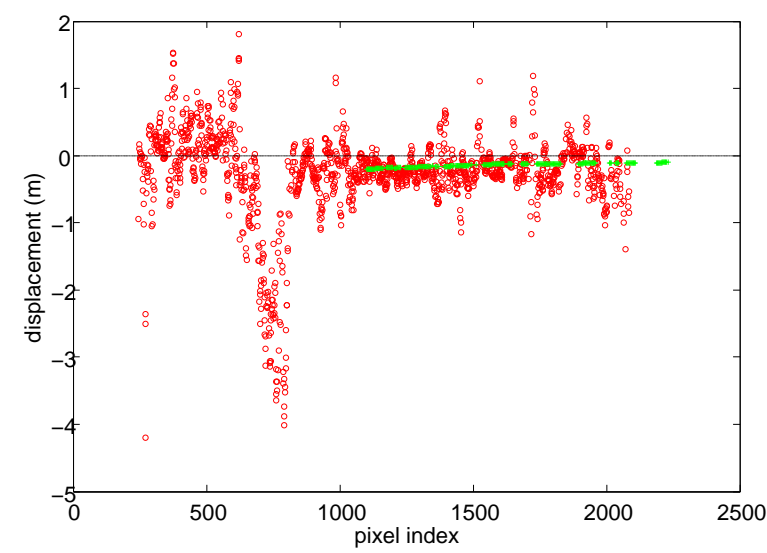

(b)

Fig. 2: Examples of displacement measurements. Red: displacements estimated by sub-pixel image correlation. Green: displacements estimated by D-InSAR

Fig. 2 shows two examples of the measurements issued from both sub-pixel image correlation and D-InSAR. The two profiles are issued from displacement images. The first profile (Fig. 2 (a)) is located in an area far from the fault and the second profile (Fig. 2 (b)) passes across the fault. In theory, the same displacement values should be found by these two techniques where both measurements are available, as they measure exactly the same quantity of displacement. However, with the presence of uncertainty in both measurements, a discrepancy of displacement values is observed on both profiles. On one hand, a more or less significant fluctuation of displacement value is observed in the sub-pixel image correlation measurements, which complies with the presence of random uncertainty. Near the fault, the fluctuation is small. While in the area far from the fault where the displacement magnitude is small, the fluctuation becomes significant. On the other hand, there is a small shift between the displace- ment values estimated by both techniques. Consequently, it is probable that systematic uncertainty is also present in one or the other measurement. However, it seems that the random uncertainty is more important than the systematic uncertainty in the considered measurements.

\section{B. 3D Displacement field retrieval by linear inversion}

The different measurements from sub-pixel image correlation and D-InSAR are different projections of the 3D displacement at the Earth's surface (E, N, Up) in the SAR image range and azimuth directions. Consequently, the $3 \mathrm{D}$ displacement field can be constructed from at least 3 different projections by a linear inversion. In this case, $R$ corresponds to different measurements from sub-pixel image correlation and D-InSAR. $P$ corresponds to the projection vectors matrix. $U$ denotes the $3 \mathrm{D}$ displacement with 3 components E, N, Up. To solve this linear inverse problem, the GLS method is used. In order to highlight the behaviours of conventional and fuzzy approaches, three levels of comparisons are considered: between displacement values, between uncertainty parameter values and between possibility distributions. Moreover, the effect of uncertainty reduction due to adding D-InSAR measurements is analyzed.

Regarding the nominal displacement value, the result obtained with D-InSAR measurements is shown in Fig. 3 (a). In fact, the results in cases with and without D-InSAR measurements are globally consistent, with an average difference in the order of millimeter. Regarding the uncertainty parameter values, $\sigma$ and $h_{0.5}, 4$ cases are considered: with and without D-InSAR measurements in both conventional and fuzzy approaches. The evolution of these values varies from one case to another (Fig. 3 (b) and (c)). Uncertainty parameter values in the fuzzy approach are always larger than those in the conventional approach. Adding measurements from DInSAR reduces the uncertainties in both conventional and fuzzy approaches. In order to understand the spatial evolution of uncertainty in both approaches, the ratio $\left(\frac{1.35 \sigma}{h_{0.5}}\right)$ of the conventional uncertainty (Fig. 3 (b)) on the fuzzy uncertainty (Fig. 3 (c)) is shown in Fig. 4. A geographic effect is observed and it corresponds to the distribution of the number of available measurements. In the darker area, the difference between the two uncertainty parameters is large, because in this area, there are more measurements available. In the conventional approach, the output uncertainty is reduced. In the fuzzy approach, on the contrary, the output uncertainty remains constant or increases slightly. Consequently, the difference between the two uncertainty parameters increases in this area.

Fig. 4 provides a summarized vision of the relationship between the two uncertainty parameters. For each point, the comparison is also perfomed between distributions: possibility distribution corresponding to the fuzzy uncertainty and equivalent possibility Gaussian distribution corresponding to the conventional uncertainty. Fig. 5 shows un example of possibility distributions for the point B. As expected, the conventional possibility distribution is more specific than the fuzzy based one. 
(a)
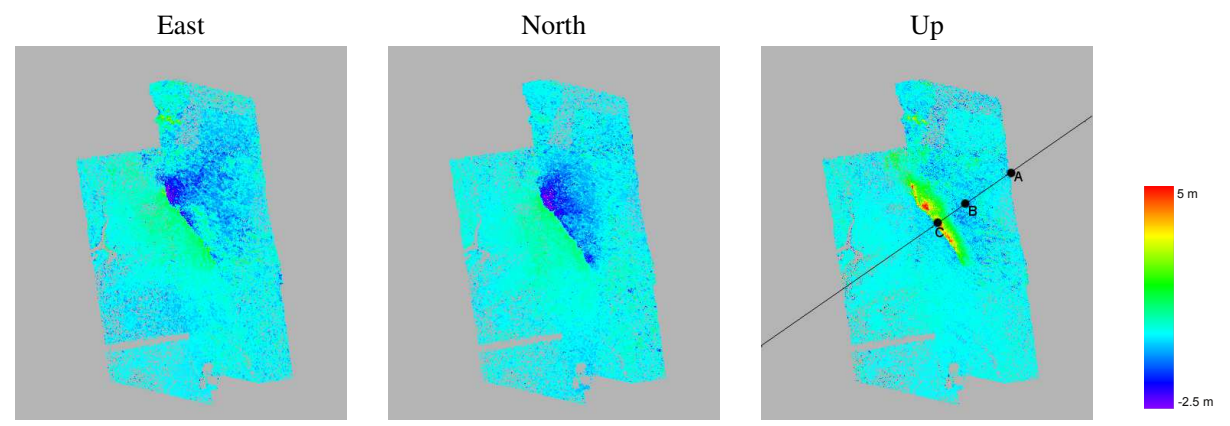

(b)
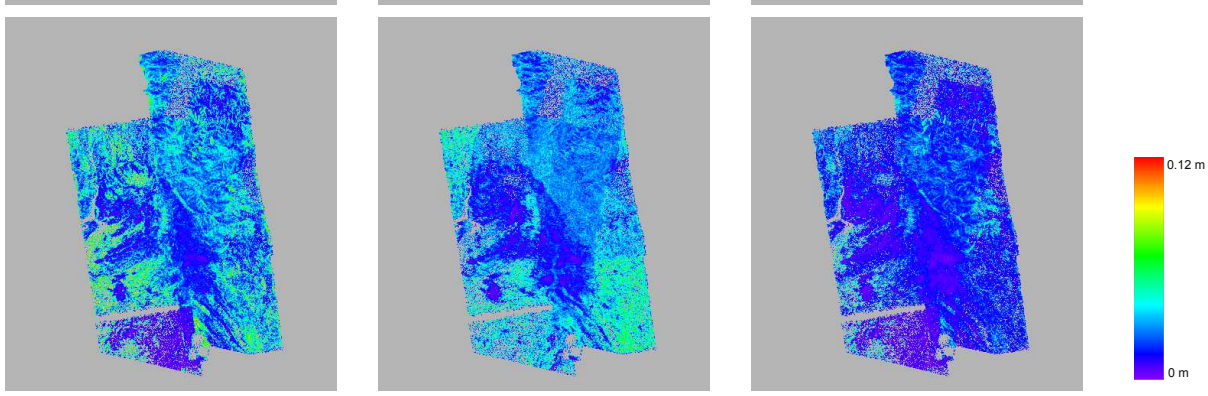

(c)
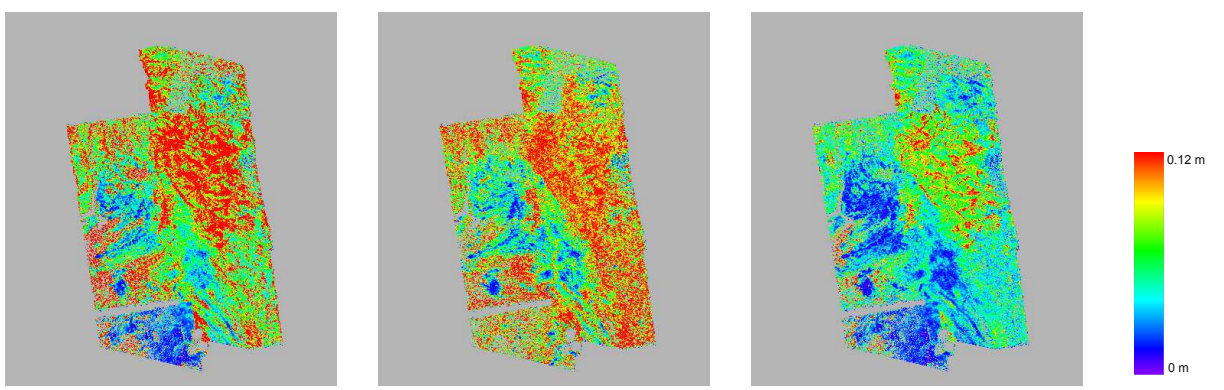

Fig. 3: (a) 3D displacement values (b) conventional uncertainty parameter values ( $\sigma$ ) (c) fuzzy uncertainty parameter values $\left(h_{0.5}\right)$ estimated in the linear case. The profile illustrated in (a) Up component, is used to compare the displacement values and uncertainties obtained in both linear and nonlinear case (Fig. 6). Point B and C are used for possibility distribution analysis (Fig. 5, Fig. 7).

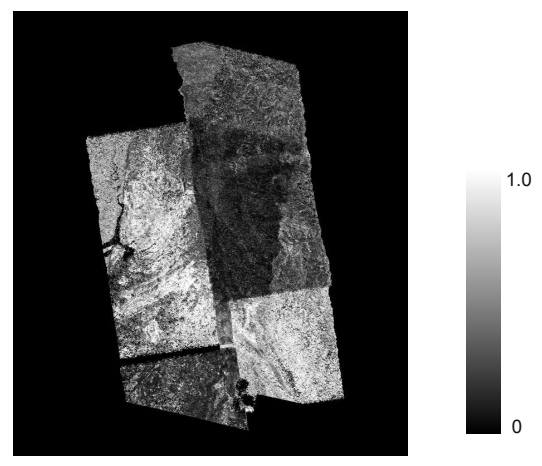

Fig. 4: Example of ratio of conventional and fuzzy uncertainty parameter values for the East component.

\section{COMPARISON WITH A MODEL BASED 3D DISPLACEMENT}

Since there is no ground truth available to validate the results obtained in the previous section, we compare them with a model based reference of the 3D displacement constructed by means of a well known mechanical deformation model.

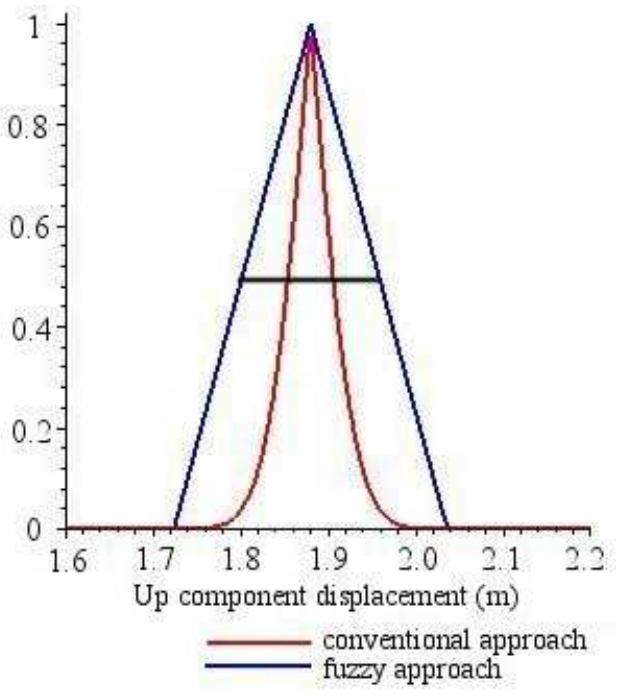

Fig. 5: Comparison of possibility distributions on point B. Blue: fuzzy approach. Red: conventional approach. The black line represents the width of the $0.5 \alpha$-cut, the uncertainty parameter used in the fuzzy approach. 
Indeed, this model is widely used since many years by geophysicists [29] [30] [31] [32] [23] [33]. Thus it is worthwhile to confront the corresponding results with the ones obtained with our approaches.

\section{A. Construction of a model based reference}

For the model based reference, the 3D displacement is deduced by 2 steps. First, the fault geometry parameters are optimised by nonlinear inversion of a mechanical deformation model describing the surface displacement field induced by a fault rupture in depth, by looking for a global minimum misfit with the surface measurements from the same input data sets as used in the previous section. At this step, the input data sets are subsampled by quadtree algorithm, thus the number of points in each measurement is greatly reduced in order not to burden the inversion. As a result, each point used in the inversion represents the deformation in an area more or less large. Second, the 3D displacement is derived from the fault geometry parameters obtained at the first step through the forward deformation model [34]. In this method, all the points used in the inversion contribute in a joint way and the fault geometry parameters correspond to a global deformation model, which is quite different from the method used in the previous section. Therefore, the displacement field obtained here is very smooth, which reveals the global deformation behaviour. By contrast, in the previous section, the neighboring points are independent, the retrieved 3D displacement reveals essentially the local deformation behaviour and is sensitive to noise.

Because of the complexity of the nonlinear inversion of the deformation model, we cannot propagate uncertainties associated with input measurements through the deformation model directly. In order to estimate the uncertainty associated with the derived 3D displacement, synthetic noise is simulated and added to the input measurements. Measurements from subpixel image correlation are mainly influenced by white gaussian noise, while measurements from D-InSAR are essentially influenced by spatially correlated noise due to atmospheric impact [4]. The characteristics of these two types of noise are estimated from preseismic data sets using both sub-pixel image correlation and D-InSAR on pairs of images without deformation. Then, 1000 simulations of white gaussian noise or spatially correlated noise with the same characteristics are carried out for each measurement according to the data type. 1000 sets of fault geometry parameters are obtained, from which 1000 values of the 3D displacement are retrieved for each pixel. Then the mean and the standard deviation are calculated, and the corresponding triangular possibility distribution is built for each pixel according to the way described in Section II-B.

The fuzzy uncertainty is used in the model based reference in order to take into account the lack of complete probability knowledge in modeling. Although the model used in this section is not perfect, it is commonly used and validated by most of the geophysicists since more than 20 years. Therefore, the results obtained in the previous section are worthwhile to be compared to the results obtained by this method.

\section{B. Comparison with the model based reference}

In order to facilitate the comparison, a profile, crossing the fault, as shown in Fig. 3 (a), is performed and the comparison is realised along this profile. The results are plotted in Fig. 6. The uncertainty ( $1.35 \sigma$ for the conventional approach, $h_{0.5}$ for the fuzzy approach and the model based reference) is here represented by uncertainty bar and the jumping of displacement value (at about $23.5 \mathrm{~km}$ from the reference point $\mathrm{A}$ ) indicates the position of the fault rupture.

According to Fig. 6, there is a good agreement between the results obtaind in the previous section and the model based reference. For most of the pixels, there is a good superposition, especially in the field far from the fault. Near the fault, for the model based reference, the maximal displacement is under estimated, which results in the shift of nominal displacement value with respect to that retrieved in the previous section. However, taking into account the uncertainty associated with the nominal displacement value, the difference is small. Thus, the good agreement, especially with the fuzzy approach, allows validating the results obtained in the previous section. It is highlighted that the uncertainty in the conventional approach is optimistic, while the uncertainty in the fuzzy approach is pessimistic but closer to the model based reference in this comparison.

Moreover, in order to make use of the richness of the possibility distribution, possibility distributions obtained in the previous section and with the model based reference are compared. Here, besides point B, we consider another point C situated on the fault (as shown in Fig. 3 (a)). The possibility distributions are shown in Fig. 7.

In Fig. 7 (a), there is a good intersection between the possibility distributions obtained in the previous section and with the model based reference. In particular, the intersection with the possibility distribution of the fuzzy approach (blue) is more significative than with the equivalent Gaussian distribution (red). Therefore, the possibility distribution of the fuzzy approach is more relevant than the equivalent Gaussian distribution. Fig. 7 (b) confirms this conclusion. In this case, the difference of results with respect to the model based reference is larger, thus there is no significative intersection with the equivalent Gaussian distribution, but a small one with the possibility distribution of the fuzzy approach.

\section{CONCLUSION}

In this article, two approaches based respectively on the probability and fuzzy theories have been applied to represent uncertainty in measurement of the 3D displacement field due to the 2005 Kashmir earthquake. The 3D displacement field is estimated by a least squares based linear inversion using the measurements from sub-pixel image correlation and DInSAR. The uncertainty is propagated in the conventional approach and in the proposed fuzzy approach respectively. The results are compared with a model based reference issued from a nonlinear inversion of a well known mechanical Earth deformation model.

According to the foundations of probability and fuzzy theories, the uncertainty in the considered probability approach 


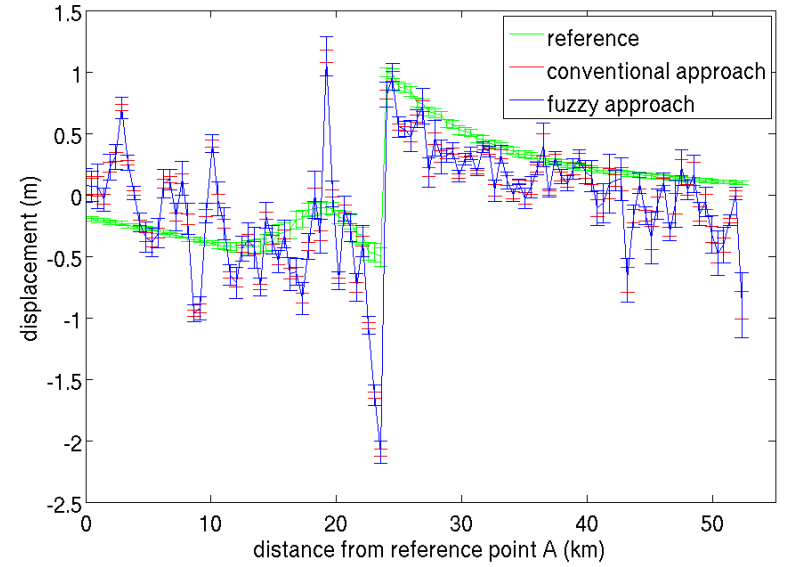

(a)

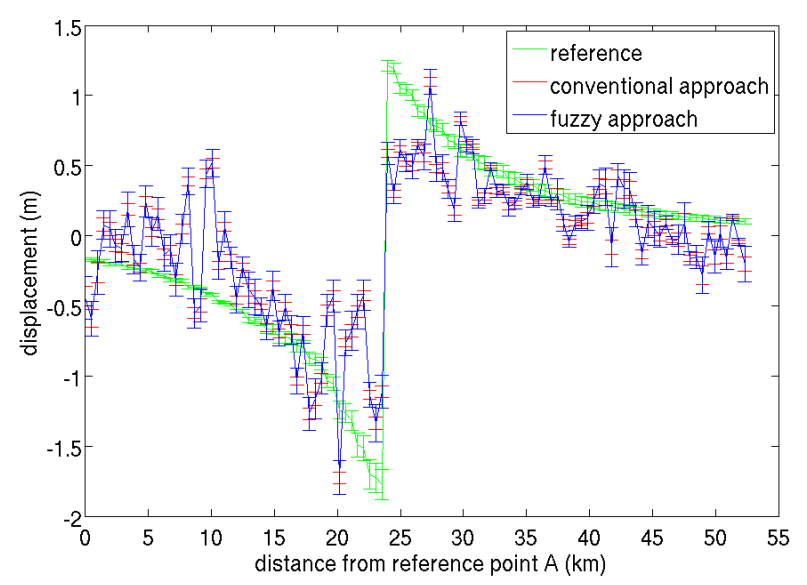

(b)

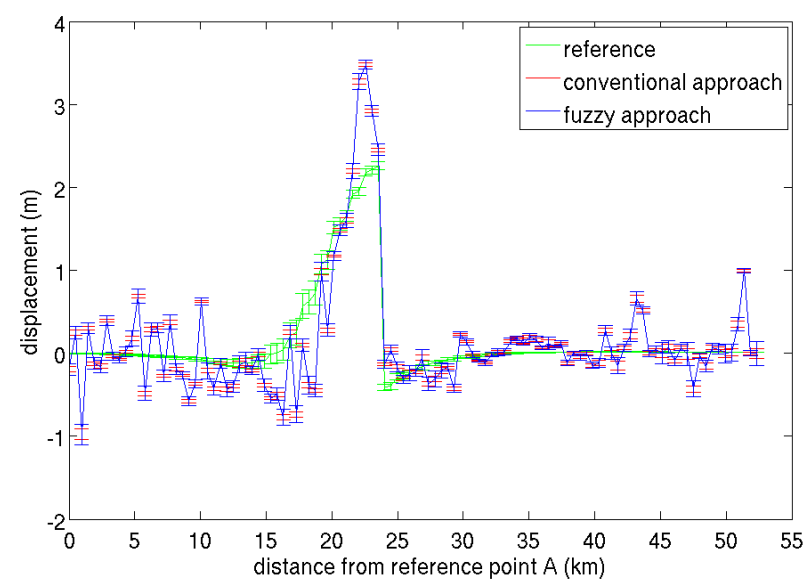

(c)

Fig. 6: Profile of displacements and uncertainties obtained by linear inversion and with the model based reference (a) East component (b) North component (c) Up component.

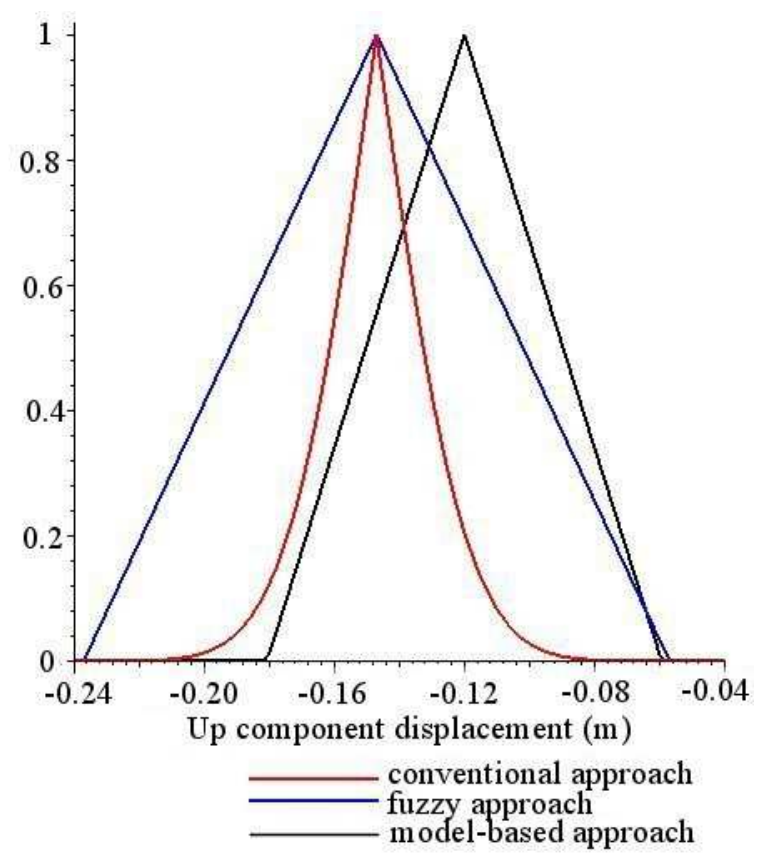

(Point B)

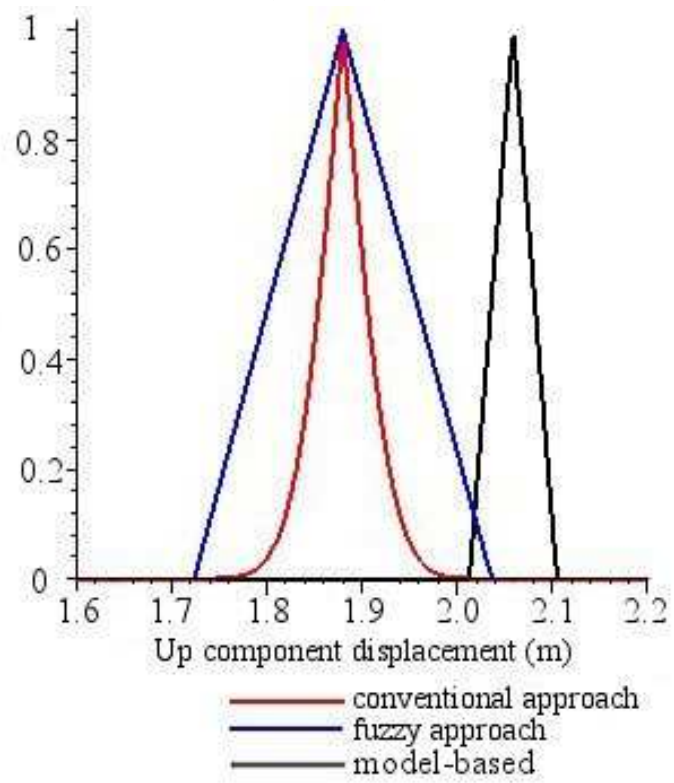

(Point C)

Fig. 7: Comparison of possibility distributions. blue: fuzzy approach. red: conventional approach. black: reference.

is under estimated, while the uncertainty in the proposed fuzzy approach is over estimated. In the context of our measurements, the actual uncertainty should be situated between these two uncertainties. The more the assumptions of Gaussian distribution and independence on uncertainties associated to input measurements, the closer to the conventional uncertainty it is. Otherwise, it is closer to the fuzzy uncertainty. Although the proposed fuzzy approach is pessimistic in uncertainty assessment, it is more robust against the assumptions of Gaussian distribution and independence on uncertainties made in the conventional approach. In fact, the fuzzy and conventional 
approaches provide respectively the most pessimistic and the most optimistic uncertainty assessment from almost similar simple analytical computations. Monte-Carlo simulations applied to the Earth deformation model provide a more relevant assessment, but with much more computational complexity and they are extremely time consuming.

In order to improve the uncertainty analysis, the part of systematic uncertainties not yet taken into account in the SAR measurements has to be identified and represented in an appropriate way. Besides this subject, the fusion of the results of the direct SAR measurements with the ones provided by the mechanical deformation model, is also a part of ongoing works.

\section{ACKNOWLEDGMENT}

This work was supported by the EFIDIR project (http://www.efidir.fr) (ANR-07-MDCO-004) and the PAKSIS program CATTELL, granted by the French National Agency (ANR). The authors wish to thank the ANR for their support. The ENVISAT data sets are available thanks to ESA project category 1 , No. 3802 .

\section{REFERENCES}

[1] E. Attema, "Mission Requirements Document for the European Radar observatory Sentinel-1," ES-RS-ESA-SY-0007, vol. 1, 2005.

[2] R. Pedersen, S. Jónsson, T. Arnadóttir, F. Sigmundsson, and K. L. Feigl, "Fault slip distribution of two June $2000 M_{w} 6.5$ earthquake in South Iceland estimated from joint inversion of InSAR and GPS measurements," Earth and Planetary Science Letters, vol. 213, no. 3-4, 2003.

[3] D. A. Schmidt, R. Bürgmann, R. M. Nadeau, and M. d'Alessio, "Distribution of aseismic slip rate on the Hayward fault inferred from seismic and geodetic data," Journal of Geophysical Research, vol. 110 no. B08406, 2005.

[4] H. Sudhaus and S. Jónsson, "Improved source modelling through combined use of InSAR and GPS under consideration of correlated data errors: application to the June 2000 Kleifarvatn earthquake, Iceland," Geophysical Journal International, vol. 176, no. 2, 2009.

[5] R. Grandin, A. Socquet, R. Binet, Y. Klinger, E. Jacques, J. de Chabalier, G. King, C. Lasserre, S. Tait, P. Tapponnier, A. Delorme, and P. Pinzuti, "September 2005 Manda Hararo-Dabbahu rifting event, Afar (Ethiopia) Constraints provided by geodetic data," Journal of Geophysical Research, vol. 114, no. B08404, 2009.

[6] G. Mauris, V. Lasserre, and L. Foulloy, "Fuzzy modeling of measurement data acquired from physical sensors," IEEE Trans. on Measurement and Instrumentation, vol. 49, no. 6, 2000.

[7] G. Mauris, "Expression of measurement uncertainty in a very limited knowledge context: a possibility theory-based approach," IEEE Trans. on Measurement and Instrumentation, vol. 56, no. 3, 2007.

[8] M. Urbanski and J. Wasowki, "Fuzzy approach to the theory of measurement inexactness," Int. Journal of Measurement, vol. 34, 2003.

[9] A. Ferrero and S. Salicone, "The random-fuzzy variables: a new approach to the expression of uncertainty," IEEE Trans. on Measurement and Instrumentation, vol. 53, no. 5, 2004.

[10] — "A comparative analysis of the statistical and random-fuzzy approaches in the expression of uncertainty in measurement," IEEE Trans. on Measurement and Instrumentation, vol. 54, no. 4, 2005.

[11] A. Mencattini and S. Salicone, "How to process the random part of RFVs: comparison of available methods and new proposals," IEEE Trans. on Measurement and Instrumentation, vol. 59, no. 1, 2010.

[12] N. Verhoest, B. D. Baets, F. Mattia, G. Satalino, C. Lucau, and P. Defourny, "A possibilistic approach to soil moisture retrieval from ERS synthetic aperture radar backscattering under soil roughness uncertainty," Water Resources Research, vol. 44, no. W06407, 2007.

[13] P. Mujumdar and S. Ghosh, "Modeling GCM and scenario uncertainty using a possibilistic approach: Application to the Mahanadi River, India," WATER RESOURCES RESEARCH, vol. 44, no. W06407, 2008.
[14] A. Jacquin, "Possibilistic uncertainty analysis of a conceptual model of snowmelt runoff," Hydrology and Earth System Sciences, vol. 14, pp. $1681-1695,2010$

[15] Y. Yan, E. Trouvé, G. Mauris, and V. Pinel, "Fuzzy vs probability uncertainty analysis of seismic displacement measurements issued from d-insar and sar image correlation measurements: Application to the kashmir earthquake (2005)," in IEEE International Instrumentation and Measurement Technology Conference, 2011.

[16] D. Dubois, L. Foulloy, G. Mauris, and H. Prade, "Probability-possibility transformations, triangular fuzzy sets and probabilistic inequalities," International Journal on Reliable Computing, vol. 10, no. 4, 2004.

[17] BIPM, IEC, IFCC, ILAC, ISO, IUPAC, IUPAP, and OIML, Evaluation of measurement data - Guide to the expression of uncertainty in measurement, JCGM 2008 ed., 2008.

[18] A. Tarantola, Inverse Problem Theory and Methods for Model Parameter Estimation. Society for Industrial and Applied Mathematics, 2005.

[19] P. Cornillon and E. Matzner-Lober, Rgression, Thorie et applications. Springer Paris, 2007

[20] L. A. Zadeh, "Fuzzy sets as a basis for a theory of possibility," Fuzzy Sets and Systems, vol. 1, no. 1, pp. 3-28, 1978.

[21] G. Mauris, V. Lasserre, and L. Foulloy, "A fuzzy approach for the expression of uncertainty in measurement," Int. Journal of Measurement, vol. 29 , no. 3, 2001.

[22] G. J. Klir and B. Yuan, Fuzzy sets and fuzzy logic: Theory and application. Prentice Hall PTR, 1995.

[23] E. Pathier, E. J. Fielding, T. J. Wright, R. Walker, B. E. Parsons, and S. Hensley, "Displacement field and slip distribution of the 2005 Kashmir earthquake from SAR imagery," Geophysical Research Letters, vol. 33, no. L20310, 2006.

[24] S. H. Yun, H. Zebker, P. Segall, A. Hooper, and M. Poland, "Interferogram formation in the presence of complex and large deformation," Geophysical Research Letters, vol. 34, no. L12305, 2007.

[25] T. J. Wright, C. Ebinger, J. Biggs, A. Ayele, G. Yirgu, D. Keir, and A. Stork, "Magma-maintained rift segmentation at continental rupture in the 2005 Afar dyking episode," Nature, vol. 442, no. 291 - 294, 2006.

[26] Davidson, Russell, Mackinnon, and J. G., Estimation and inference in econometrics, isbn 978-0-19-506011-9 ed. Oxford University Press, 1993.

[27] P. Rosen, S. Hensley, G. Peltzer, and M. Simons, "Updated Repeat Orbit Interferometry Package released," Transactions, American Geophysical Union, vol. 85, 2004

[28] E. Trouvé, J. Nicolas, and H. Maitre, "Improving Phase Unwrapping Techniques by the Use of Local Frequency Estimates," IEEE Transaction on Geoscience and Remote Sensing, vol. 36, no. 6, 1998.

[29] P. Clarke, D. Paradissis, P. Briole, P. England, B. Parsons, H. Billiris, G. Veis, and J. Ruegg, "Geodetic investigation of the 13 May 1993 Kozani - Grevena (Greece) earthquake," Geophysical Research Letters, vol. 24, no. 6, 1997.

[30] T. Wright, B. Parsons, J. Jackson, M. Haynes, E. Fielding, P. England, and P. Clarke, "Source parameters of the 1 October 1995 Dinar (Turkey) earthquake from SAR interferometry and seismic body wave modelling," Earth and Planetary Science Letters, vol. 172, 1999.

[31] S. Jónsson, H. Zebker, P. Segall, and F. Amelung, "Fault Slip Distribution of the $1999 M_{w} 7.1$ Hector Mine, California, Earthquake, Estimated from Satellite Radar and GPS Measurements," Bulletin of the Seismological Society of America, vol. 92, no. 4, 2002.

[32] G. J. Funning, B. Parsons, and T. J. Wright, "Surface displacements and source parameters of the 2003 Bam (Iran) earthquake from Envisat advanced synthetic aperture radar imagery," Journal of Geophysical Research, vol. 110, no. B09406, 2005.

[33] S. Atzori, I. Hunstad, M. Chini, S. Salvi, C. Tolomei, C. Bignami, S. Stramondo, E. Trasatti, A. Antonioli, and E. Boschi, "Finite fault inversion of DInSAR coseismic displacement of the 2009 L'Aquila earthquake (central Italy)," Geophysical Research Letters, vol. 36, no. L15305, 2009.

[34] Y. Okada, "Surface deformation due to shear and tensile faults in a halfspace," Bulletin of the Seismological Society of America, vol. 75, no. 4, 1985. 